BMC

Developmental Biology

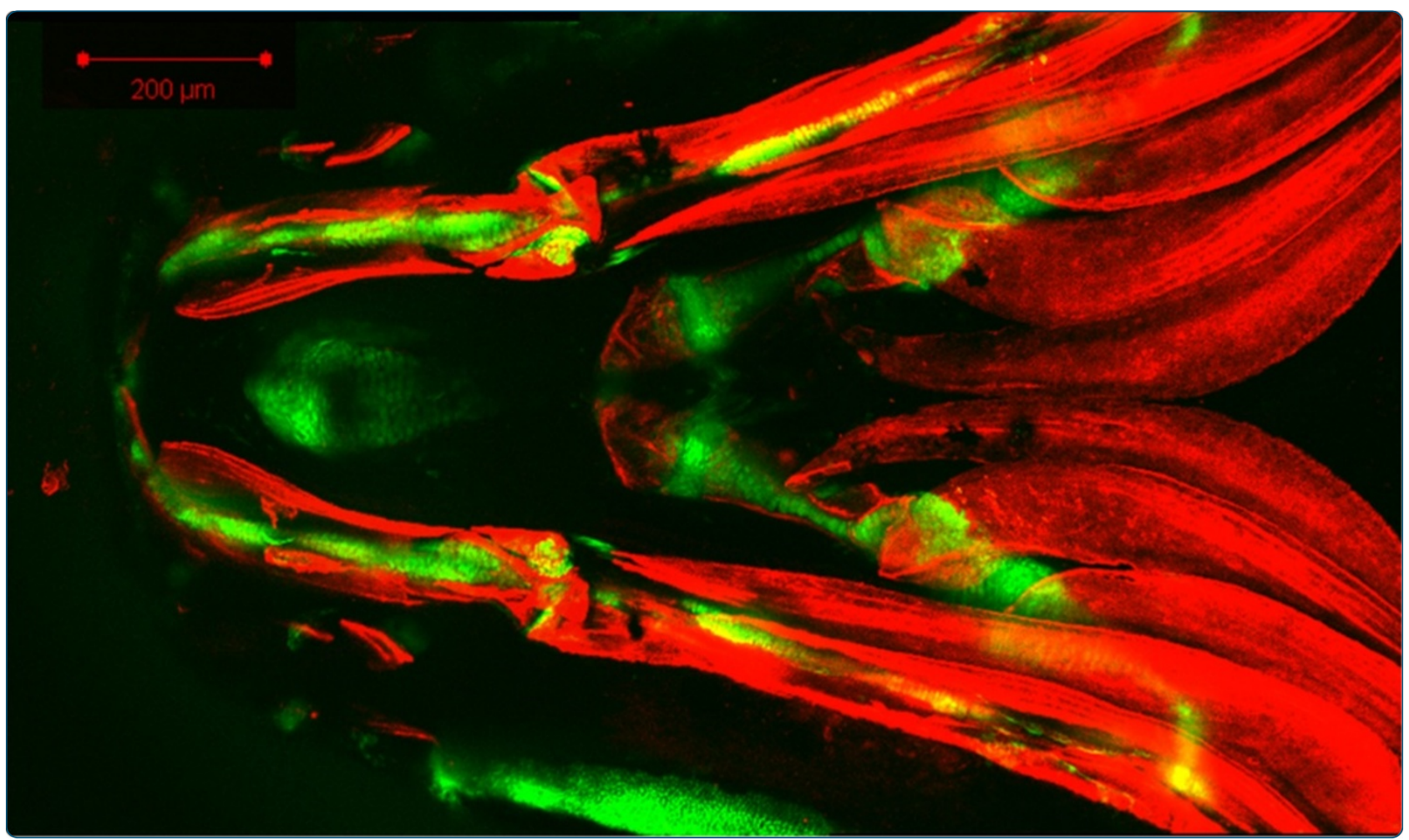

FishFace: interactive atlas of zebrafish craniofacial development at cellular resolution

Eames et al.

() Biomed Central 


\title{
FishFace: interactive atlas of zebrafish craniofacial development at cellular resolution
}

\author{
B Frank Eames ${ }^{1,2^{*}}$, April DeLaurier ${ }^{1 *}$, Bonnie Ullmann ${ }^{1}$, Tyler R Huycke ${ }^{1,3}$, James T Nichols ${ }^{1}$, John Dowd ${ }^{1}$, \\ Marcie McFadden ${ }^{1}$, Mark M Sasaki ${ }^{1,4}$ and Charles B Kimmel ${ }^{1 *}$
}

\begin{abstract}
Background: The vertebrate craniofacial skeleton may exhibit anatomical complexity and diversity, but its genesis and evolution can be understood through careful dissection of developmental programs at cellular resolution. Resources are lacking that include introductory overviews of skeletal anatomy coupled with descriptions of craniofacial development at cellular resolution. In addition to providing analytical guidelines for other studies, such an atlas would suggest cellular mechanisms underlying development.

Description: We present the Fish Face Atlas, an online, 3D-interactive atlas of craniofacial development in the zebrafish Danio rerio. Alizarin red-stained skulls scanned by fluorescent optical projection tomography and segmented into individual elements provide a resource for understanding the 3D structure of the zebrafish craniofacial skeleton. These data provide the user an anatomical entry point to confocal images of Alizarin redstained zebrafish with transgenically-labelled pharyngeal arch ectomesenchyme, chondrocytes, and osteoblasts, which illustrate the appearance, morphogenesis, and growth of the mandibular and hyoid cartilages and bones, as viewed in live, anesthetized zebrafish during embryonic and larval development. Confocal image stacks at high magnification during the same stages provide cellular detail and suggest developmental and evolutionary hypotheses.
\end{abstract}

Conclusion: The FishFace Atlas is a novel learning tool for understanding craniofacial skeletal development, and can serve as a reference for a variety of studies, including comparative and mutational analyses.

Keywords: Craniofacial development, Website atlas, Zebrafish, Bone, Cartilage, Skeleton, Evolution

\section{Background}

How do elements of the vertebrate craniofacial skeleton arise, grow, and reshape during development, and how do these processes vary during the course of evolution? Answers to these questions are coming from both moleculargenetic and cell-biological approaches, which rely, first of all, on precise description of the developmental events and processes that comprise skeletogenesis. The availability of developmental atlases facilitates such study. Indeed, many descriptive atlases of craniofacial development in various vertebrate species have been published [1-4]. In today's world, internet atlases are extremely useful, in part

\footnotetext{
* Correspondence:b.frank@usask.ca; april@uoneuro.uoregon.edu; kimmel@uoneuro.uoregon.edu

${ }^{1}$ Institute of Neuroscience, University of Oregon, Eugene, OR, USA ${ }^{2}$ Current address: Department of Anatomy \& Cell Biology, University of Saskatchewan, Saskatoon, SK, Canada

Full list of author information is available at the end of the article
}

because they are publicly accessible around the world, but also because they can be formatted so that a user interacts with the data in unique ways, such as with freely-rotatable 3D models (e.g., Mouse Limb Atlas: http://www.nimr.mrc. ac.uk/3dlimb/; [5]).

Zebrafish, with patterns of development conserved among all vertebrates, with favorable attributes for phenotypic analyses of development, and with a sophisticated knowledge by the research community of its genetics and genomics, provides a powerful animal model for learning about craniofacial development. Online zebrafish atlases include the Zebrafish Atlas (zfatlas.psu.edu); 3D Atlas of Zebrafish Vasculature Anatomy (http://uvo.nichd.nih.gov/ atlas.html); the Zebrafish Brain Atlas (http://www.ucl.ac. uk/zebrafish-group/zebrafishbrain/index.php); the Atlas of Zebrafish Anatomy (http://www.zebrafish.uni-freiburg.de/ anatomy.html); the Atlas of Zebrafish Development (http://bio-imaging.liacs.nl/liacsatlas.html); the Zebrafish

\section{Ciomed Central}


Anatomy Portal (http://www.zfap.org); and the FishNet 3D developmental atlas (http://www.fishnet.org.au/index. shtml). None of these atlases, however, currently focus on development of the craniofacial skeleton.

Zebrafish craniofacial development can be detailed with an investigative method unsurpassed in accuracy and sensitivity. Using current transgenic approaches in combination with vital dye staining, one can examine craniofacial skeletal elements in exquisite cellular detail during an extended period of development within living, intact zebrafish embryos and larvae. Here, we describe a high-resolution developmental atlas of the zebrafish craniofacial skeleton, the FishFace Atlas (https://www.facebase.org/fishface/home), to serve as a guide for anatomical and cellular studies of craniofacial development.

\section{Utility}

\section{Summary of FishFace Atlas website organization}

The FishFace Atlas focusses on cellular level anatomy of the developing skeleton in the first two pharyngeal arches. It uses confocal imaging of living specimens. To assist the user unfamiliar with fish craniofacial anatomy, FishFace also provides an overview of the structure of the skull, featuring optical projection tomography (OPT) of preserved material. In this "Skull Anatomy" section, fluorescent OPT images of Alizarin red-stained samples show skull bones of middle and late larval, and adult, zebrafish (https://www.facebase. org/fishface/opt_data; for clarification of the relationship between the FishFace Atlas and FaceBase hub, please see (Additional file 1)). Furthermore, a model of larval craniofacial bones was created by segmentation of 3D reconstructed OPT data, and this model can be freely rotated and virtually dissected in a user-defined fashion (https://www.facebase. org/fishface/Viewer).

The "Arch 1 and 2 Development" section of FishFace complements data from "Skull Anatomy", including 'overview' series of low magnification confocal image stacks through the two most anterior pharyngeal arches and through the craniofacial skeleton derived from these arches. These images show transgenically-labelled cells in living embryos and larvae, and all ontological terms in their descriptions link directly to standardized definitions on the Zebrafish Information Network (http://zfin.org/). One such 'overview' is a series of embryonic zebrafish at preskeletal stages showing transgenically-labelled neural crest-derived mesenchymal cells that eventually give rise to craniofacial skeletal elements (https://www.facebase. org/fishface/early_arches). The second 'overview' series illustrates developing skeletal elements using transgenicallylabelled chondrocytes and Alizarin-red stained bone matrix (https://www.facebase.org/fishface/arch1_and_2).

The heart of the FishFace Atlas is the "Element Development" section, which uses high magnification confocal image stacks of transgenically-labelled chondrocytes or osteoblasts, along with Alizarin red-stained mineralized bone matrix, to offer insight into the genesis of the anatomical complexity demonstrated by the OPT and low magnification 'overview' confocal data. This 'element development' section adds cellular detail to selected skeletal elements in pharyngeal arch one and pharyngeal arch two, tracking ontogenetic sequences of the following individual cartilages and bones as they develop during embryonic and larval stages:

- palatoquadrate (https://www.facebase.org/fishface/ palatoquadrate);

- Meckel's cartilage (https://www.facebase.org/ fishface/meckel);

- hyosymplectic (https://www.facebase.org/fishface/ hyosymplectic);

- ceratohyal (https://www.facebase.org/fishface/ ceratohyal); and

- opercle (https://www.facebase.org/fishface/ op_and_bsr_series).

By studying elements in cellular detail, a much more precise description can be made of the dramatic changes to element growth and shaping that occur in early developmental stages. Most images in FishFace have links to movies playing slice by slice through each confocal stack of the z-series that was used to generate that particular confocal projection. Hence, the FishFace Atlas provides the community with a valuable, interactive resource with which the user can understand not only the complex 3D anatomical relationships of skeletal elements, but also the underlying cellular organization, in the developing zebrafish craniofacial skeleton.

\section{Skull Anatomy: an interactive model of zebrafish craniofacial skeletal anatomy}

To provide an understanding of the overall anatomy of the zebrafish craniofacial skeleton, we used fluorescent optical projection tomography (OPT) imaging of Alizarin red-stained adult, 18 days post-fertilization ( $\mathrm{dpf}$ ), and 14 dpf zebrafish heads. The adult zebrafish head has a beautifully complex assortment of 74 bones covering almost the entire dorsal, lateral, and ventral surfaces (Figure 1A, B; [6]). A general theme of the FishFace Atlas is to facilitate understanding of such complexity by tracking structures earlier in development, when the anatomy is simpler. By $14 \mathrm{dpf}$, for example, OPT imaging demonstrates that the craniofacial skeleton of the zebrafish consists principally of a small number of lateral and ventral bones in the pharyngeal arches (Figure $1 \mathrm{C}, \mathrm{D}$ ). To reveal the complex relationships of the many bones of the zebrafish craniofacial skeleton, the user may view movies that cycle through the raw data of the initial scans, which take an image from each of 400 rotational angles of the specimen (e.g., links 


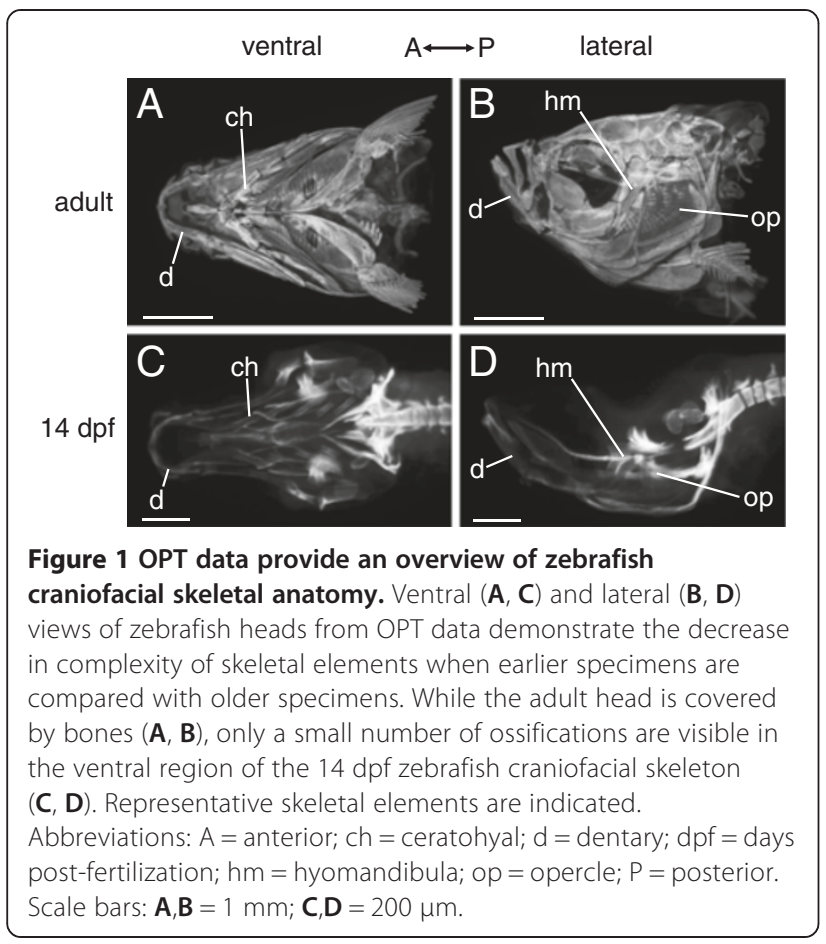

on https://www.facebase.org/fishface/opt_data). Downloadable movies on this webpage (please see "OPT imaging and processing" within 'Construction and content') also enable the user to progress from anterior to posterior through 3D reconstructions of the OPT data.

Finally, we created from the $14 \mathrm{dpf} 3 \mathrm{D}$ reconstructions a model of zebrafish skeletal anatomy, which the user can visualize through the JAtlas Viewer program (https://www. facebase.org/fishface/Viewer). In this highly-interactive format, the user can build, rotate, magnify, and virtually dissect elements of the juvenile zebrafish craniofacial skeleton (Figure 2A). Individual bones are color-coded and annotated, and users manipulate 3D models and generate virtual 2D sections through the specimen (Figure 2B, C). Using the 'mouse-click anatomy' in the section viewer (under Show/mouse-click anatomy), users can use their mouse to click on a feature in the 2D section view, and the name of the element appears at the top of the viewer window. We anticipate that $2 \mathrm{D}$ views will be helpful especially for users seeking annotation tools while interpreting histological data.

\section{Arch 1 and 2 Development: arch ectomesenchyme to cartilages and bones}

To understand development of the first two pharyngeal arches, mandibular and hyoid, the FishFace Atlas uses confocal imaging in living preparations. Generated from transgenic zebrafish, the images illustrate cellular details not only of the developing pharyngeal arches, but also of the cartilages and bones that form within them. As such, the confocal data provide a nice complement to the OPT data, which visualizes mineralized matrix of the developing zebrafish craniofacial skeleton. Two sets of lowmagnification confocal images help the user transition from the OPT dataset and show how craniofacial morphology changes with time. One covers six stages before, and the other eight stages after, skeletal elements become individually recognizable. Both generally include ventral and lateral views.

Selected lateral views from FishFace show how zebrafish craniofacial morphology undergoes remarkable changes during embryonic and larval periods (Figure 3). Embryonic pharyngeal arches (Figure 3A), made largely from neural crest-derived ectomesenchyme expressing fli1a:EGFP, appear relatively homogeneous and simply organized at 32 hpf (even though developmental patterning studies show that they are anything but homogeneous and simple at this stage [7]). About a day later (Figure 3B, $55 \mathrm{hpf}$ ), expression of sox9a:EGFP reveals early cartilage rudiments developing in these arches. Two cartilages, one dorsal and one ventral, have formed in each arch on each side of the body. No bones are yet mineralized. FishFace then illustrates how this early skeletal pattern progressively elaborates and increases in complexity, such that by about midway through larval development (Figure 3C, 14 dpf) the morphology looks completely different from earlier stages.

\section{Element Development: detailed single-cell resolution of imaging suggests developmental and evolutionary hypotheses of individual skeletal elements}

The final component of the FishFace Atlas, and the most novel, is a set of 'element' pages. This resource includes abundant images at high magnification that provide cellular resolution to accompany the lower magnification 'overview' images just discussed. By using the 'overview' and 'element' parts of the atlas together, the viewer is able to directly connect developing element morphologies with changes in cellular patterning, such as cellular arrangements, shapes, and sizes. Together, these help to elucidate the cellular basis of morphogenesis and growth. Here we use the mandible, the lower jaw, to show that imaging with cellular resolution allows one to support hypotheses that then can be tested by more directed and analytically based study. The mandible represents an interesting case study, due to its complex developmental and evolutionary history [1,8-10].

The first mandibular skeletal element is Meckel's cartilage, present by $55 \mathrm{hpf}$ (Figure 4A). During the following days, the cartilage grows in size and reshapes, becoming longer and relatively thinner (Figure 4, compare A and B; and view the many more images showing Meckel's cartilage in the Fishface Atlas under "Element Development"). Cells might mediate this kind of shape change, known as convergence and extension, by one or more of several distinct activities; they might reshape, migrate, and/or intercalate with 


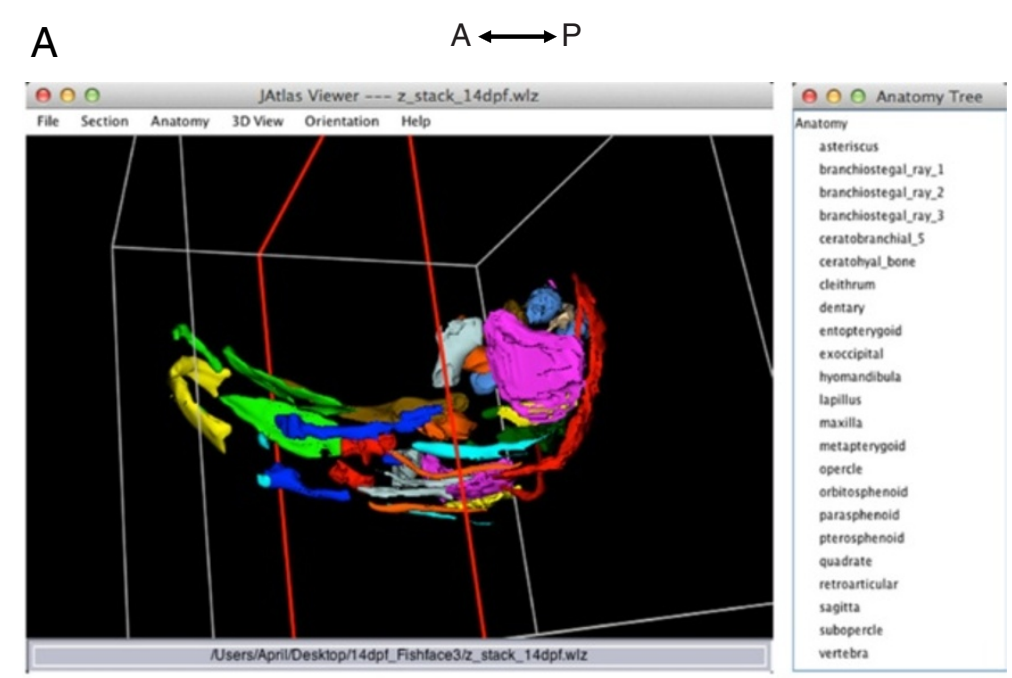

\section{B}

C

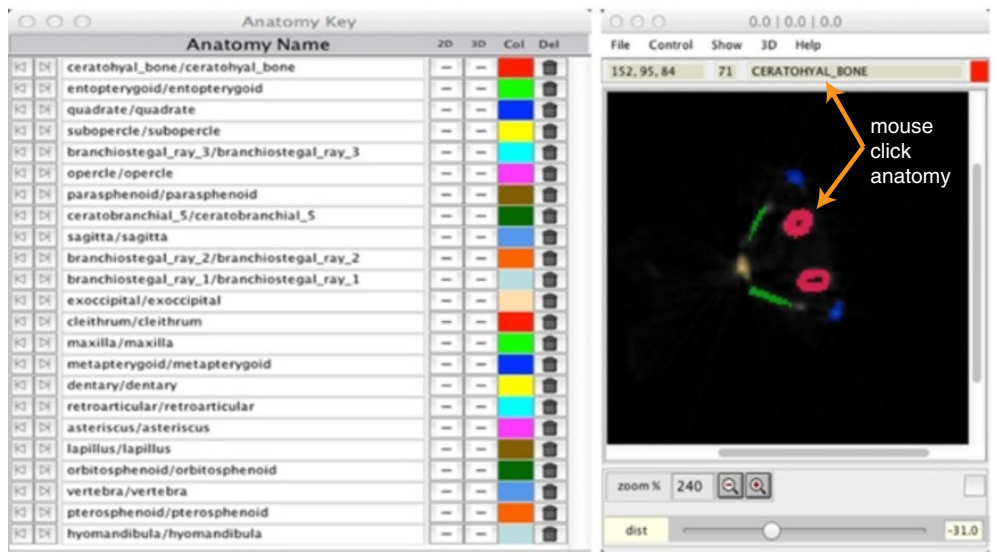

Figure 2 Skeletal anatomy of the zebrafish head at $14 \mathrm{dpf}$ visualized using Jatlas Viewer. The interactive 3D model of skeletal structures (A) can be rotated in a user-defined fashion with a click and drag of the mouse. The Anatomy Tree (right) lists all of the structures displayed in the 3D view. The Anatomy Key (B) shows the name of each structure in the 3D model and its color code. Structures can be switched on and off in the 2D and 3D viewer windows by clicking on "+/-" in the 2D and 3D columns of the Anatomy Key. Structures can be removed and replaced from the 3D and 2D viewers by clicking on the icon in the Del column. Colors can be changed by clicking in the Col column, which opens a palette of colors to choose from. In the 2D section view $(\mathbf{C})$, structures shown in the $3 \mathrm{D}$ viewer are displayed in the same colors. With the 'mouse-click anatomy' function, users can use their mouse to click structures on the 2D section, and the name appears at the top of the 2D viewer window. The plane of section is shown as a red outline in the 3D model (A), and can be changed by adjusting 'yaw' and 'pitch'.

one another. Detailed analyses show that cellular intercalation is the predominant cellular basis of notochord convergence and extension [11,12], and the FishFace images motivate intercalation as a leading hypothesis to explain convergence and extension of Meckel's cartilage as well. At early stages (Figure 4A), the chondrocytes are in a seemingly disordered arrangement, generally more than two of them spanning the mediolateral thickness of Meckel's cartilage. Within two days (Figure 4B), a longer, thinner cellular array has become more ordered, with long stretches of the cartilage comprised of a one-cell wide stack.

Cellular imaging also provides support for hypotheses explaining growth in size of individual cartilages. As is well known, chondrocytes greatly enlarge in size, especially during early stages of differentiation morphogenesis, and the number of chondrocytes in growing cartilages increases as well (e.g., as quantified for the zebrafish symplectic cartilage [4]). New chondrocytes can come from the perichondrium (resulting in appositional growth), and they can come from cell divisions of the chondrocytes themselves (resulting in interstitial growth). Interstitial growth yields distinctive chondrocyte doublets, which at first occupy single lacunae within the matrix. FishFace images support interstitial growth of Meckel's cartilage (but do not exclude appositional growth). Compared to arrangements of chondrocytes and their separating matrix in Meckel's 

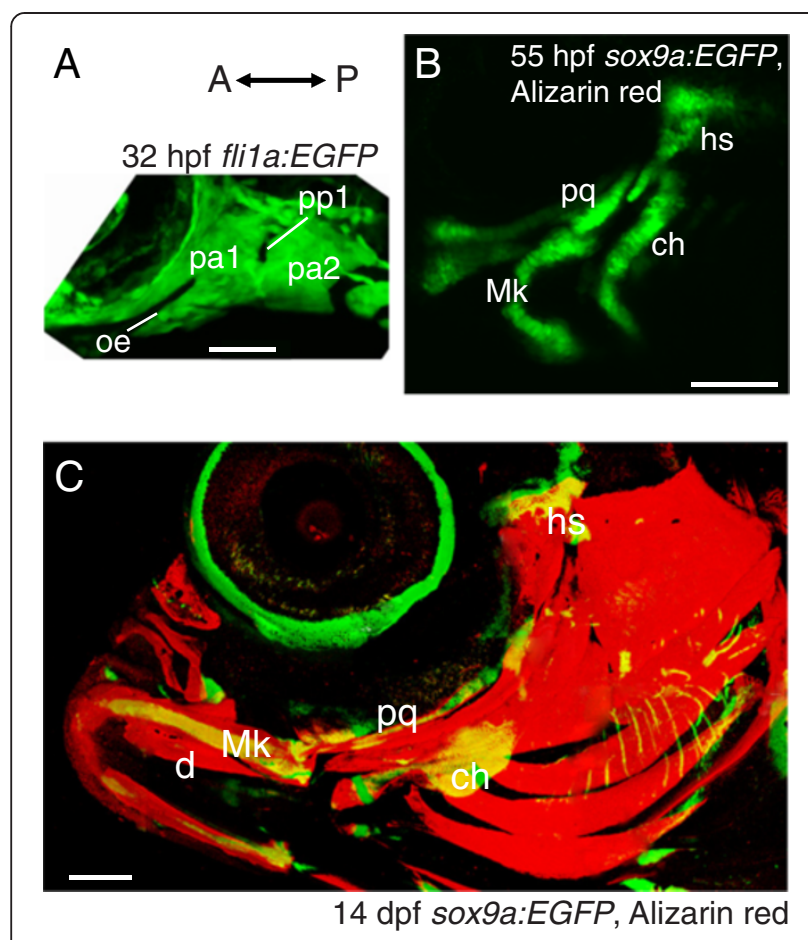

Figure 3 Low magnification confocal images demonstrate dynamics of morphology and complexity during zebrafish craniofacial development. Lateral view of fli a:EGFP zebrafish at 32 hpf (A) illustrates apparent simple and homogenous arrangement of cells in the anterior two pharyngeal arches. Lateral view of Alizarin redstained sox9a:EGFP zebrafish at $55 \mathrm{hpf}$ (B) demonstrates that most cartilages, including dorsal and ventral elements, have begun to form in these pharyngeal arches, while no bones are visible. Compared to $55 \mathrm{hpf}$, Alizarin red-stained sox9a:EGFP zebrafish by $14 \mathrm{dpf}$ (C) show that cartilage elements have changed in morphology and size, but the addition of many bones results in a major increase in complexity. Abbreviations: $\mathrm{A}=$ anterior; $\mathrm{ch}=$ ceratohyal; $\mathrm{d}=$ dentary; $\mathrm{dpf}=$ days post-fertilization; $\mathrm{hpf}=$ hours post-fertilization; hs = hyosymplectic; $\mathrm{Mk}=$ Meckel's cartilage; oe $=$ oral ectoderm; $\mathrm{P}=$ posterior;

$\mathrm{pa}=$ pharyngeal arch; $\mathrm{pp}=$ pharyngeal pouch; $\mathrm{pq}=$ palatoquadrate. Scale bars: $\mathbf{A}=50 \mu \mathrm{m} ; \mathbf{B}=100 \mu \mathrm{m} ; \mathbf{C}=200 \mu \mathrm{m}$.

cartilage at $4 \mathrm{dpf}$ (Figure $4 \mathrm{C}, \mathrm{C}^{\prime}$ ), images at $6 \mathrm{dpf}$ capture what seems to be a nest of doublets (Figure 4D,D'; essentially all of the cells in Figure 4D' are included in this nest), and suggest that cell-cycles are locally synchronous.

Besides developmental hypotheses, such as the two just proposed for cartilage morphogenesis and growth, the precision afforded by live confocal imaging at cellular resolution can motivate and support evolutionary hypotheses. For example, Jollie regards as a teleost synapomorphy that the most prominent bone in the mandible, the dermal dentary, is actually compound, whereby its anterior end is comprised of a chondral element, the mentomeckelian [13]. As evidence, some species (e.g., Esox, pike; Salmo, salmon) have a separate mentomeckelian in larvae, which then becomes fused with the dentary [14]. However, imaging in the FishFace Atlas suggests a different mechanism in zebrafish, leading to the hypothesis that a single compound (i.e., fused) element is present from the earliest stages of bone formation. At $3 \mathrm{dpf}$, before any matrix is detected by the sensitive vital Alizarin red staining, only one local cluster, not two, of early osteoblasts is apparent using the sp7:EGFP transgenic zebrafish (Figure 5A). After mineralization ( $4 \mathrm{dpf}$ and later), only a single Alizarin redlabelled bone is detected (Figure 5B), but labelling of skeletogenic mesenchyme with fli1a:EGFP zebrafish demonstrate that two regions of the bone have different associations with the underlying chondrocytes. Anteriorly, the bone lies directly adjacent to the chondrocytes in the manner of a chondral bone (the mentomeckelian; yellow arrow, Figure $5 \mathrm{~B})$. More posteriorly, the bone appears to pass through the perichondrium to lie superficial to it, with transgenic cells visible between the bone and the chondrocytes, in the manner of a dermal bone (the dentary; majenta arrow, Figure $5 \mathrm{~B}$ ). If supported by definitive analyses, this zebrafish condition of the mentomeckelian fusing with the dentary from its earliest stages would provide new evidence of how bones might be 'lost' during evolution, widespread among vertebrates, including humans (see Discussion).

We emphasize that the Fishface images by themselves motivate, but do not establish, the validity of the above hypotheses. All three hypotheses we propose here are amenable to rigorous testing. For example, in time-lapse recordings of appropriate strains of transgenic zebrafish, one can observe directly, in the intact fish, cell intercalations mediating convergence and extension [11], waves of cell division [15], and whether osteoblasts are initially recruited to two adjacent bone primordia that quickly coalesce, or to a single one [16].

\section{Discussion}

The FishFace Atlas is an interactive, 3D tool for understanding aspects of the complex anatomy of the developing zebrafish craniofacial skeleton at cellular resolution. FishFace builds on and elucidates the foundation set out primarily in Cubbage and Mabee (1996), who comprehensively described skeletal elements in the zebrafish skull from fixed preparations stained for cartilage and bone through adult stages, but also in other studies that included cartilage development [17] and more detailed investigations of both cartilage [4] and bone [16,18] morphogenesis and growth. FishFace first provides overviews of the whole skull and then focusses on the first and second pharyngeal arches, the region that probably has been the most thoroughly examined region of the zebrafish skull, and which promises to continue to yield fundamental information on its genetics, patterning, morphogenesis and growth [7]. Therefore, the FishFace Atlas should be particularly useful in comparative and mutational analyses where there is interest in understanding not only the genetic basis, but also the cellular basis of skeletogenesis and patterning. 


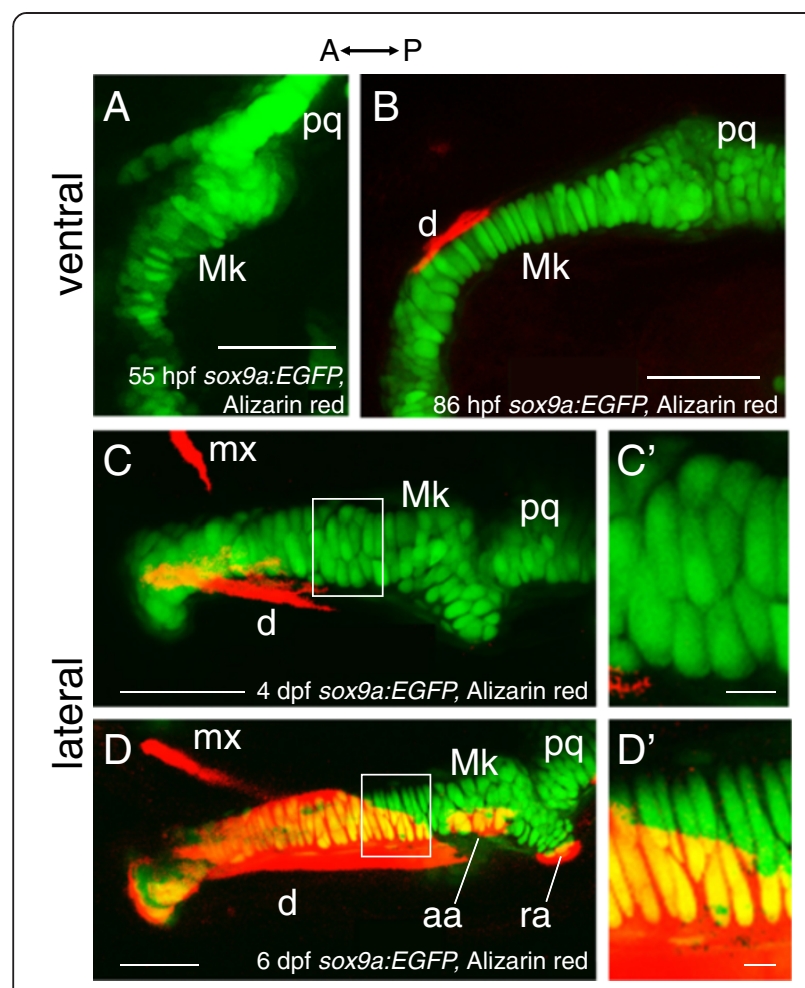

Figure 4 Chondrocyte intercalation and proliferation seem to drive cartilage growth in late embryonic and early larval stages. Confocal images in ventral view of Alizarin red-stained sox9a:EGFP mandibles suggest that early phases of Meckel's cartilage morphogenesis may be achieved by chondrocyte intercalation. By 55 hpf (A), chondrocytes in Meckel's cartilage do not appear in ordered rows, as generally two to three cells span the mediolateral width of the element. By 86 hpf (B), Meckel's cartilage has grown in length and is relatively thinner from that seen at $55 \mathrm{hpf}$, and many of its chondrocytes now span the mediolateral width in a cellular stack. Confocal images in lateral view of Alizarin red-stained sox9a: EGFP mandibles suggest that later phases of Meckel's cartilage morphogenesis may be achieved by chondrocyte proliferation. Chondrocytes of Meckel's cartilage are separated clearly from each other by $4 \mathrm{dpf}$ (C). By $6 \mathrm{dpf}$, proliferation of chondrocytes about mid-way along the anterior-posterior length of Meckel's cartilage (D) is suggested by the presence of chondrocyte doublets. These doublets are separated from each other by a layer of presumed extracellular matrix that appears much thinner than that observed between the doublets. $\mathbf{C}^{\prime}$ and $\mathbf{D}^{\prime}$ show high magnification views of the boxed regions in $\mathbf{C}$ and $\mathbf{D}$, respectively. Abbreviations:

$\mathrm{A}=$ anterior; $\mathrm{aa}=$ anguloarticular; $\mathrm{d}=$ dentary; $\mathrm{dpf}=$ days postfertilization; $\mathrm{hpf}=$ hours post-fertilization; $\mathrm{Mk}=$ Meckel's cartilage; $\mathrm{mx}=$ maxilla; $\mathrm{P}=$ posterior; $\mathrm{pq}=$ palatoquadrate; $r \mathrm{a}=$ retroarticular Scale bars: $\mathbf{A}-\mathbf{D}=50 \mu \mathrm{m} ; \mathbf{C}^{\prime}, \mathbf{D}^{\prime}=5 \mu \mathrm{m}$.

The imaging resolution of skeletal elements in the FishFace Atlas illuminates not only cellular behaviors that drive early craniofacial morphogenesis in living embryos and larvae, but also potential evolutionary mechanisms. Previous studies of craniofacial development have shown that cell migration, aggregation, intercalation, proliferation, enlargement, recruitment, and death, all play crucial roles in generating proper morphology $[4,16,19-22]$. As we point out for the mandible, the FishFace images support developmental hypotheses explaining reshaping and organized growth of its cartilaginous element, Meckel's cartilage. Cellular intercalations may drive convergence and extension, and interstitial growth, perhaps including mitotic waves, might contribute to growth. The images also support a compound nature of the dentary, a posterior dermal element fused with an anterior chondral one. We hypothesize that the fusion is present in the osteoblast primordium (i.e., condensation) that initially makes the bone, which is consistent with a mechanism of skeletal evolution expounded by Atchley and Hall [23]. Furthermore, Patterson, in his important review of skeletal element evolution, proposes that often bone 'loss' is actually fusion and may occur in evolutionary sequences [24]. The primitive condition is multiple bones: two in the case of anterior mandibular bones, with independent mentomeckelian and dentary bones apparent in teleost outgroups, such as the bowfin Amia. Then a step occurs that Patterson calls "ontogenetic fusion", in which separate bone rudiments are present in early ontogeny and then fuse (as for the trout and pike; [14]). Finally, as a yet more derived condition, there is "phylogenetic fusion" in Patterson's words; where the ancestor had two (or more) bones, the descendant at all developmental stages has but a single one. Patterson notes that convincing evidence for phylogenetic fusion is lacking [24]. As pointed out above, time-lapse studies in zebrafish could provide this evidence, an analysis motivated by imaging in the FishFace Atlas.

\section{Conclusions}

In order to increase understanding of the development of the zebrafish craniofacial skeleton at cellular resolution, the FishFace Atlas is a website that presents images generated by confocal microscopy and optical projection tomography (OPT) in a user-interactive format. We anticipate its utility as a reference for mutational and evolutionary analyses.

\section{Construction and Content}

\section{Larval rearing and quality control}

All zebrafish lines were maintained, and zebrafish embryos and larvae were raised, according to established protocols $[4,25]$ with IACUC approval (see http://zfin.org/zf_info/ zfbook/chapt3/3.2.html). Briefly, up to 20 larvae in $150 \mathrm{ml}$ EM were fed paramecia in $250 \mathrm{ml}$ beakers from 4 days post-fertilization (dpf) to $9 \mathrm{dpf}$, when they were shifted to mouse cages and fed paramecia and baby brine shrimp until $15 \mathrm{dpf}$, when they were fed only brine shrimp. Regarding quality control of larval rearing, co-authors followed their strict published methods [18] to monitor general hallmarks of larval health (e.g., swim bladder, prey capture) in our nursery, and standard length measurements were taken for each imaged specimen. Regarding 

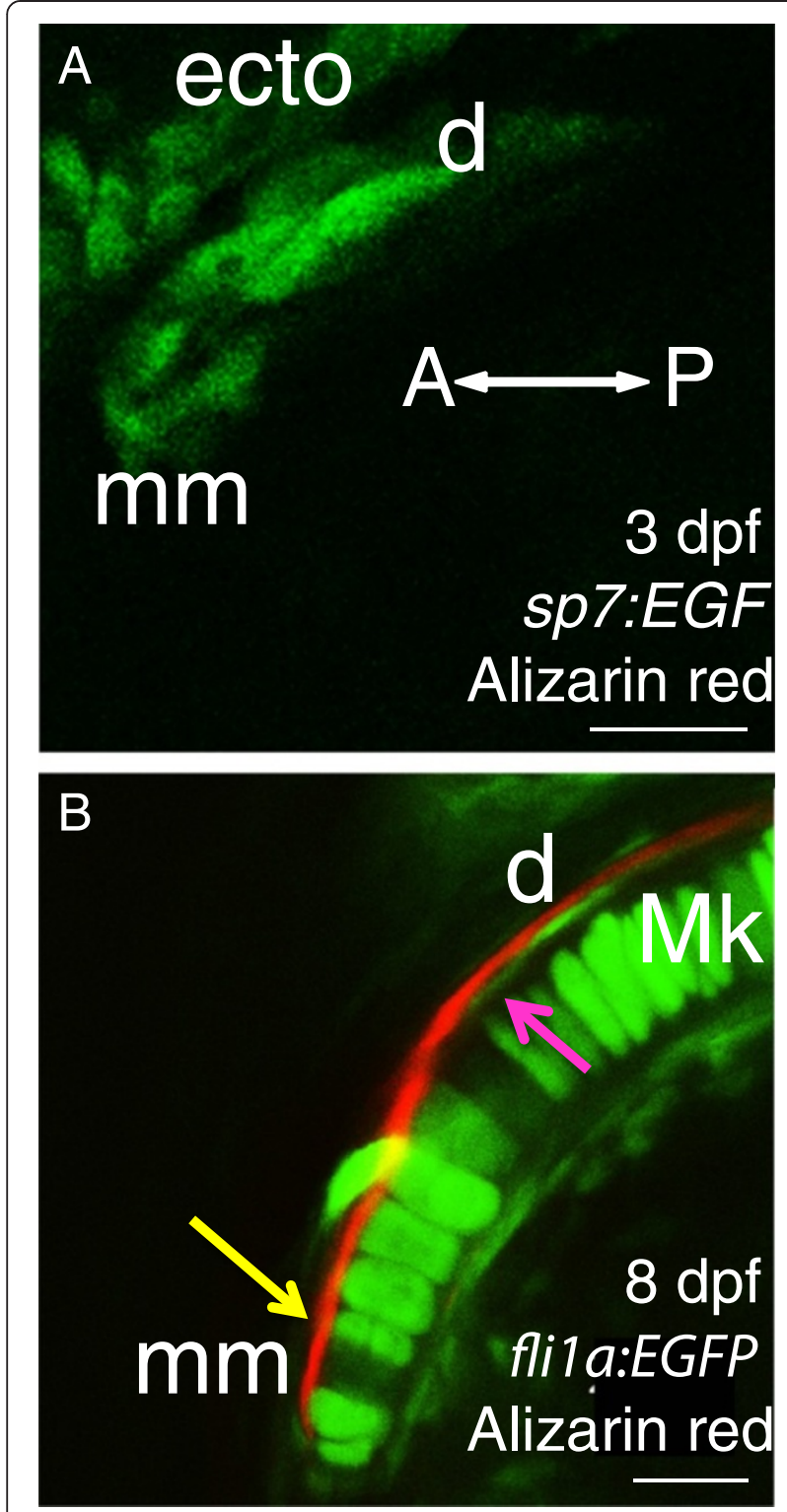

Figure 5 Cellular resolution of bones in the anterior Meckel's cartilage suggests that the dentary has fused with the mentomeckelian bone from the earliest stages of osteogenesis. Before bone matrix is detected by Alizarin red, only one group of osteoblasts is apparent adjacent to Meckel's cartilage in $3 \mathrm{dpf}$ Alizarin red-stained sp7:EGFP larvae from a ventral view (A). In a confocal slice of the anterior region of Meckel's cartilage in $8 \mathrm{dpf}$ Alizarin red-stained flita: EGFP larvae (B), a single bone is visible from a ventral view, but its association with chondrocytes of Meckel's cartilage appears to vary anteroposteriorly. Anteriorly, bone lies immediately adjacent to chondrocytes (yellow arrow), while more posteriorly, the bone appears to be separated from chondrocytes by cells of the presumptive perichondrium (magenta arrow).

Abbreviations: $\mathrm{A}=$ anterior; $\mathrm{d}=$ dentary; $\mathrm{dpf}=$ days post-fertilization; ecto = ectoderm; Mk = Meckel's cartilage; $\mathrm{mm}=$ mentomeckelian; $\mathrm{P}=$ posterior. Scale bars: $\mathbf{A}, \mathbf{B}=20 \mu \mathrm{m}$. quality control of images included in FishFace, co-authors of the paper collected the images, which were selected for Atlas inclusion by consensus among co-authors, based upon 1) how well the images reflected average development of multiple specimens, often from multiple independent clutches; and 2) how consistent the data were with relevant published literature (e.g., $[4,6,17])$.

\section{Alizarin red staining}

For OPT imaging of mineralized matrix, fixed zebrafish specimens were stained as described previously [26]. Tissues were fixed overnight in 4\% PFA, washed for an hour in $1 \% \mathrm{KOH}$, bleached in $3 \% \mathrm{H}_{2} \mathrm{O}_{2} / 0.5 \% \mathrm{KOH}$ for $40 \mathrm{~min}$. with lids open, washed in $1 \% \mathrm{KOH}$, stained overnight in $0.003 \%$ Alizarin red in $1 \% \mathrm{KOH}$, and de-stained in $1 \%$ $\mathrm{KOH}$. After eyes were removed, heads were rinsed in water, embedded in low melting point agarose (Invitrogen), washed twice in methanol, and cleared in benzyl alcohol: benzyl benzoate (1:2). For confocal imaging of mineralized matrix in live specimens, zebrafish larvae were maintained in Embryo Medium supplemented with $0.005 \%$ Alizarin red and 0.01 M HEPES at least two hours, and often overnight, prior to confocal imaging [18].

\section{OPT imaging and processing}

Images of craniofacial bones of $14 \mathrm{dpf}, 18 \mathrm{dpf}$, and adult zebrafish were captured using Bioptonics OPT Scanner 3001 M (MRC Technology). 3D reconstructions of raw data were made using NRecon (MRC Technology). Segmentations of skeletal elements in reconstructions were made using Amira 5.2.2 (Visage Imaging), and movies highlighting specific skeletal elements were created in Amira and QuickTime Player. Movies showing progression through virtual sections of the reconstruction were created in QuickTime Player (Apple). For information on how to download movies from FishFace for importation into ImageJ, please see the Additional file 1.

\section{Transgenic animals}

All transgenic fish were maintained on the $\mathrm{AB}$ background.

The transgenic zebrafish line $\operatorname{sox} 9 a^{z c 81 T g}$, which we refer to here as sox9a:EGFP, was created using Gateway technology and includes a portion of the foxp 2 promoter driving EGFP expression ( $\operatorname{Tg}(f o x p 2 . A: E G F P)$; [27]). Please see Construction and content and Additional file 2 for details on how sox9a:EGFP was isolated from the transgenic line $\operatorname{Tg}($ foxp2.A:EGFP) zc42 [27].

The transgenic zebrafish line $\operatorname{Tg}(s p 7: E G F P) b 1212$, which we refer to here as $s p 7: E G F P$, was created using BAC transgenesis and includes a large portion of the $s p 7$ promoter driving EGFP expression [28].

The transgenic zebrafish line $\operatorname{Tg}(f l i 1 a: E G F P) y 1$, which we refer to here as fli1a:EGFP, was created by microinjecting a construct including a portion of the fli1a 
promoter driving EGFP expression [29] and has been used widely as a marker of neural crest cells and their derivatives, as well as of endothelial cells (e.g., [19,30,31]).

\section{Cloning genomic locus of sox $9 a^{\text {zc81Tg }}$}

Genomic DNA was isolated from fins of three adult sox $9 a^{z c 81 T g}$, which we refer to here as sox9a:EGFP, zebrafish with cartilage expression and of one non-glowing sibling adult zebrafish (QIAGEN DNeasy, QIAGEN Inc.). $1 \mu \mathrm{g}$ gDNA from each of these four samples was digested with AluI or HaeIII in $40 \mu \mathrm{l}$ at $37 \mathrm{C}$ for 3 hours. $0.5 \mu \mathrm{g}$ of digested gDNA was ligated with $4 \mu \mathrm{l}$ T4 DNA ligase (Epicentre) in $500 \mu \mathrm{l}$ overnight at $16 \mathrm{C}$. The reaction was concentrated using Zymo-5 column (Zymo Research Corp.) and resuspended in $20 \mu \mathrm{l}$ for use as PCR template. Inverse PCR was carried out according to published protocols for cloning Tol2 insertion sites into the zebrafish genome [32,33]. First-round PCR primers were either [Tol2-3'invf2 + Tol23'/r1] or [Tol2-3'invf1 + Tol2-3'invr1], while second-round PCR primers were either [Tol2-3'/f2 + Tol2-3'invr2] or [Tol2-3'invf2 + Tol2-3'invr2] [32,33]. No PCR products were obtained from non-transgenic fish. Many clones from products of the different PCR primer pairs were sequenced from each transgenic fish, and sequences BLASTed to the same location (Chr12: 2278311; Zv9, www.ensembl.org).

\section{Confocal microscopy}

Imaging of live zebrafish specimens at various time points on $1,2,3,4,6,8,10,14$, and $21 \mathrm{dpf}$ was conducted using either a Zeiss LSM 5 Pascal confocal or Leica SD6000 spinning disk confocal with Borealis illumination technology. In order to visualize Alizarin red staining with most sensitivity, pinhole and/or detector gain settings were adjusted by hand to levels just below those that showed red fluorescence in surrounding, non-mineralized tissues. Maximum projections were made from stacks of images that demonstrated the entire depth of the element(s) under focus. Movies showing progression through all images of the stack were created in Pascal (Carl Zeiss, Inc.) or Metamorph (Leica Microsystems) and sometimes were edited in ImageJ (NIH) for orientation and cropping.

\section{Comments on developmental generalizations}

The FishFace Atlas illustrates a generalized developmental sequence of the appearance of specific skeletal elements, but there are caveats to such generalizations. First, development is a dynamic, variable process. Apart from time-lapse movies, temporal series of images are merely snapshots of this process and thus cannot represent the full dynamics of developmental processes. Second, considerable clutch variation exists within a given "wild-type" stock, let alone between "wild-type" stocks under use around the world. We have attempted to normalize such differences to some extent by noting the standard length of each specimen and also by comparing morphological features of specimens to standardized staging series [34,35]. In addition, we tried to select specimens that were representative of the clutch at each timepoint. Finally, imaging always is subject to limits of detection, so we try to avoid making declarative statements that a given skeletal element "appears" at a given time. That said, we have refined our methods to detect skeletal elements with the most sensitive techniques available. Our confocal imaging illustrates specifically skeletogenic cells prior to their secretion of abundant extracellular matrix. Also, we incubate and image our specimens with the vital dye Alizarin red while they are alive. As such, we avoid the severe reduction in Alizarin red binding that occurs after even brief periods in fixative and artificial buffers, for these chemicals leech mineral from skeletal tissues in the specimen. In summary, the FishFace Atlas addresses potential pitfalls of a variable developmental process and limitations of skeletal detection to provide the user an understanding of development of the pharyngeal arches and its skeleton in the embryonic and larval zebrafish.

\section{Availability and requirements}

The data sets supporting the results of this article are included within the article (and its Additional files 1 and 2) or are available in the FishFace Atlas (https://www. facebase.org/fishface/home). There are no restrictions on use by non-academics. All users are free to download images or movies (please see Additional file 1).

\section{Additional files}

Additional file 1: Relationship between the FishFace Atlas and
FaceBase hub, Mechanism for adding material to FishFace,
Instructions for downloading FishFace data.
Additional file 2: Isolation and genomic cloning of a chondrocyte-
specific transgenic line, sox9a ${ }^{\text {zc81Tg }}$, from Tg(foxp2.A:EGFP)zc42. We
have reported previously sp7:EGFP (formally called Tg(sp7:EGFP)b1212;
[28]), a zebrafish line that illuminates osteoblast formation and
distribution during bone development, but we needed its counterpart in
cartilage development. Here, we characterize a novel transgenic insertion
that has chondrocyte-specific expression. Tg(foxp2.A:EGFP)zc42 was
reported to have two bright domains of expression: 1) brain and 2)
pharyngeal arches (Additional file 2 : Figure S1A; [27]). The brain
expression was expected from the known foxp2 expression patterns, but
the pharyngeal arch expression was unexpected, as foxp2 is not
expressed in this tissue [27]. Fish were isolated with bright expression
domains in either brain or pharyngeal arches (Additional file 2: Figure
S1B, Figure S1C), suggesting that at least two insertions of the Gateway
construct were responsible for the two initial transgene expression
domains. For the following reasons, we hypothesized that expression of
the Tg(foxp2.A:EGFP) construct in pharyngeal arches was due to position-
dependent genomic effects, similar to an enhancer trap [36]. Only the
brain, and not the pharyngeal arch, expression domain of Tg(foxp2.A:
EGFP)zc42 could be recapitulated by injecting the Tg(foxp2.A:EGFP)
construct into fertilized eggs (data not shown). Moreover, there was only
one initial founder of Tg(foxp2.A:EGFP)zc42 with the pharyngeal arch
expression (J. Bonkowsky, pers. comm.). To test further the enhancer-trap
hypothesis, we used zebrafish with only the pharyngeal arch expression
domain (Additional file 2: Figure S1C) to identify the genomic locus of


the $\operatorname{Tg}($ foxp2.A:EGFP) insertion (see Construction and content). Inverse PCR revealed that the insertion site is linked to the known chondrocyte differentiation gene sox9a, approximately $120 \mathrm{~kb}$ upstream of the sox9a transcriptional start site in a $400 \mathrm{~kb}$ stretch of the genome devoid of known genes (Additional file 2: Figure S1D; see also Construction and content). Indeed, analyses of later-staged zebrafish larvae demonstrated that the pharyngeal arch expression was specific to developing chondrocytes (Figures 2, 3 and 4). Interestingly, the transgene inserted very close to an approximately $300 \mathrm{bp}$ non-coding sequence that is conserved among medaka, stickleback, fugu, frog, mouse, and human (http://genome. ucsc.edu/cgi-bin/hgTracks?db=danRer7\&position=Chr12:2153000-2307118), Due to its genetic linkage, the approved formal name of this transgenic line is sox9 $a^{\text {ze8itg }}$ (www.zfin.org), which we will refer to hereafter as sox9a:EGFP.

\section{Abbreviations}

Anatomical: Terminology and abbreviations used in the FishFace Atlas are based largely upon Cubbage and Mabee (1996) and Zebrafish Information Network ontology (http://zfin.org).

\section{Competing interests}

The authors declare that they have no competing interests.

\section{Authors' contributions}

$A D$ and $C B K$ conceived of the study. $M M$ and JD raised the fish. BFE, $A D, B U$, $\mathrm{TRH}$, JTN, and MMS performed the imaging. BFE and CBK wrote the manuscript. All authors read and approved the final manuscript.

\section{Acknowledgements}

We thank members of the FaceBase hub for establishing and maintaining the FishFace Atlas on their website; Josh Bonkowsky and the laboratory of the late Chi-Bin Chien for providing Tg(foxp2.A:EGFP)zc42, in which they noticed unexpected pharyngeal arch transgene expression; all members of the University of Oregon Fish Facility; and Richard Baldock, Bill Hill, and Nicholas Burton from the Edinburgh Mouse Atlas Project (EMAP), MRC Human Genetics Project for their generous assistance with converting OPT data so it could be viewed in JatlasViewer. This work was supported by $\mathrm{NIH}$ grants F32DE019345 to AD, F32GM086027 to JTN, F32DE019056 to MMS, and RC1DE020655, R01DE13834, and PO1HD22486 to CBK.

\section{Author details}

${ }^{1}$ Institute of Neuroscience, University of Oregon, Eugene, OR, USA. ${ }^{2}$ Current address: Department of Anatomy \& Cell Biology, University of Saskatchewan, Saskatoon, SK, Canada. ${ }^{3}$ Current address: Biological and Biomedical Sciences, Harvard University, Cambridge, MA, UK. ${ }^{4}$ Current address: Committee on Cancer Biology, University of Chicago, Chicago, IL, USA.

Received: 13 December 2012 Accepted: 21 May 2013 Published: 28 May 2013

\section{References}

1. de Beer GR: The Development of the Vertebrate Skull. Chicago: University of Chicago Press; 1937:554.

2. Bellairs R, Osmond M: The atlas of chick development. Amsterdam; Boston; London: Elsevier; 2005:470. xxiv.

3. Kaufman MH: The atlas of mouse development. London; San Diego: Academic Press; 1992:512. xvi.

4. Kimmel CB, Miller CT, Kruze G, Ullmann B, BreMiller RA, et al: The shaping of pharyngeal cartilages during early development of the zebrafish. Dev Biol 1998, 203:245-263.

5. Delaurier A, Burton N, Bennett M, Baldock R, Davidson D, et al: The Mouse Limb Anatomy Atlas: an interactive 3D tool for studying embryonic limb patterning. BMC Dev Biol 2008, 8:83.

6. Cubbage CC, Mabee PM: Development of the cranium and paired fins in the zebrafish, Danio rerio (Ostariophysi, Cyprinidae). J Morphol 1996, 229:121-160.

7. Medeiros DM, Crump JG: New perspectives on pharyngeal dorsoventral patterning in development and evolution of the vertebrate jaw. Dev Biol 2012, 371:121-135.

8. Janvier P: Early vertebrates. Oxford: Oxford University Press; 1996:393.
9. Kuratani S: Evolution of the vertebrate jaw: comparative embryology and molecular developmental biology reveal the factors behind evolutionary novelty. J Anat 2004, 205:335-347.

10. Kuratani S, Nobusada Y, Horigome N, Shigetani Y: Embryology of the lamprey and evolution of the vertebrate jaw: insights from molecular and developmental perspectives. Philos Trans R Soc Lond B Biol Sci 2001, 356:1615-1632.

11. Glickman NS, Kimmel CB, Jones MA, Adams RJ: Shaping the zebrafish notochord. Development 2003, 130:873-887.

12. Keller R, Davidson L, Edlund A, Elul T, Ezin M, et al: Mechanisms of convergence and extension by cell intercalation. Philos Trans $R$ Soc Lond $B$ Biol Sci 2000, 355:897-922.

13. Jollie M: A primer of bone names for the understanding of the actinopterygian head and pectoral girdle skeletons. Canadian Journal of Zoology 1986, 64:365-379.

14. Pehrson $\mathrm{T}$ : The development of the latero-sensory canal bones in the skull of Esox lucius. Acta Zoologica (Stockholm) 1944, 25:134-157.

15. Keller PJ, Schmidt AD, Wittbrodt J, Stelzer EH: Reconstruction of zebrafish early embryonic development by scanned light sheet microscopy. Science 2008, 322:1065-1069.

16. Huycke TR, Eames BF, Kimmel CB: Hedgehog-dependent proliferation drives modular growth during morphogenesis of a dermal bone. Development 2012, 139:2371-2380.

17. Schilling TF, Kimmel CB: Musculoskeletal patterning in the pharyngeal segments of the zebrafish embryo. Development 1997, 124:2945-2960.

18. Kimmel CB, DeLaurier A, Ullmann B, Dowd J, McFadden M: Modes of developmental outgrowth and shaping of a craniofacial bone in zebrafish. PLoS One 2010, 5:e9475.

19. Eberhart JK, He X, Swartz ME, Yan YL, Song H, et al: MicroRNA Mirn140 modulates Pdgf signaling during palatogenesis. Nat Genet 2008, 40:290-298.

20. Hall BK, Miyake T: The membranous skeleton: the role of cell condensations in vertebrate skeletogenesis. Anatomy and Embryology 1992, 186:107-124.

21. Eames BF, Schneider RA: The genesis of cartilage size and shape during development and evolution. Development 2008, 135:3947-3958.

22. Graham A, Koentges G, Lumsden A: Neural crest apoptosis and the establishment of craniofacial pattern: an honorable death. Mol Cell Neurosci 1996, 8:76-83.

23. Atchley WR, Hall BK: A model for development and evolution of complex morphological structures. Biological Reviews of the Cambridge Philosophical Society 1991, 66:101-157.

24. Patterson C: Cartilage bones, dermal bones, and membrane bones, or the exoskeleton versus the endoskeleton. In Problems in Vertebrate Evolution. Edited by Andrews S, Miles R, Walker A. London: Academic Press; 1977:77-121.

25. Westerfield M: The zebrafish book, A guide for the laboratory use of zebrafish (Danio rerio). Eugene: Univ. of Oregon Press; 2007

26. Eames BF, Yan YL, Swartz ME, Levic DS, Knapik EW, et al: Mutations in fam $20 \mathrm{~b}$ and xylt 1 reveal that cartilage matrix controls timing of endochondral ossification by inhibiting chondrocyte maturation. PLOS Genet 2011, 7:e1002246.

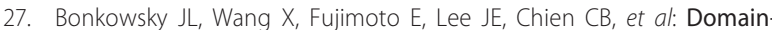
specific regulation of foxP2 CNS expression by lef1. BMC Dev Biol 2008, 8:103.

28. DeLaurier A, Eames BF, Blanco-Sanchez B, Peng G, He X, et al: Zebrafish sp7:EGFP: a transgenic for studying otic vesicle formation, skeletogenesis, and bone regeneration. Genesis 2010, 48:505-511.

29. Roman BL, Pham VN, Lawson ND, Kulik M, Childs S, et al: Disruption of acvrl1 increases endothelial cell number in zebrafish cranial vessels. Development 2002, 129:3009-3019.

30. Das A, Crump JG: Bmps and id2a act upstream of Twist1 to restrict ectomesenchyme potential of the cranial neural crest. PLoS Genet 2012, 8:e1002710.

31. LeClair EE, Topczewski J: Development and regeneration of the zebrafish maxillary barbel: a novel study system for vertebrate tissue growth and repair. PLoS One 2010, 5:e8737.

32. Kawakami K, Takeda H, Kawakami N, Kobayashi M, Matsuda N, et al: A transposon-mediated gene trap approach identifies developmentally regulated genes in zebrafish. Dev Cell 2004, 7:133-144. 
33. Kotani T, Nagayoshi S, Urasaki A, Kawakami K: Transposon-mediated gene trapping in zebrafish. Methods 2006, 39:199-206.

34. Kimmel CB, Ballard WW, Kimmel SR, Ullmann B, Schilling TF: Stages of embryonic development of the zebrafish. Dev Dyn 1995, 203:253-310

35. Parichy DM, Elizondo MR, Mills MG, Gordon TN, Engeszer RE: Normal table of postembryonic zebrafish development: staging by externally visible anatomy of the living fish. Dev Dyn 2009, 238:2975-3015.

36. Scott EK, Mason L, Arrenberg AB, Ziv L, Gosse NJ, et al: Targeting neural circuitry in zebrafish using GAL4 enhancer trapping. Nat Methods 2007, 4:323-326

doi:10.1186/1471-213X-13-23

Cite this article as: Eames et al:: FishFace: interactive atlas of zebrafish craniofacial development at cellular resolution. BMC Developmental Biology 2013 13:23.

\section{Submit your next manuscript to BioMed Central and take full advantage of:}

- Convenient online submission

- Thorough peer review

- No space constraints or color figure charges

- Immediate publication on acceptance

- Inclusion in PubMed, CAS, Scopus and Google Scholar

- Research which is freely available for redistribution 